\title{
Patients record systems: effects on dental practice and patient oral health outcomes (Protocol)
}

Nasser M, Fedorowicz Z, Newton T, Van Weel C, van Binsbergen JJ, Van de Laar FA

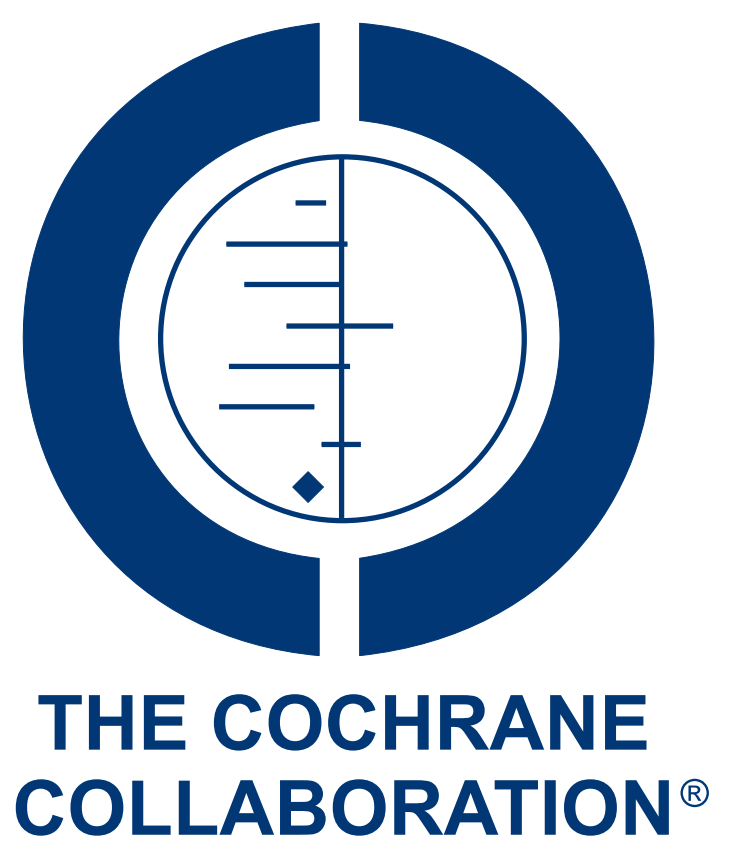

This is a reprint of a Cochrane protocol, prepared and maintained by The Cochrane Collaboration and published in The Cochrane Library 2010, Issue 7

http://www.thecochranelibrary.com

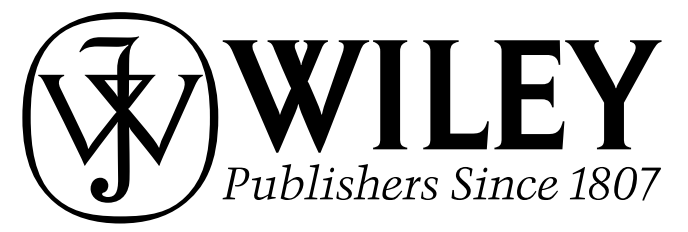

Patients record systems: effects on dental practice and patient oral health outcomes (Protocol)

Copyright @ 2010 The Cochrane Collaboration. Published by John Wiley \& Sons, Ltd. 
TABLE OF CONTENTS

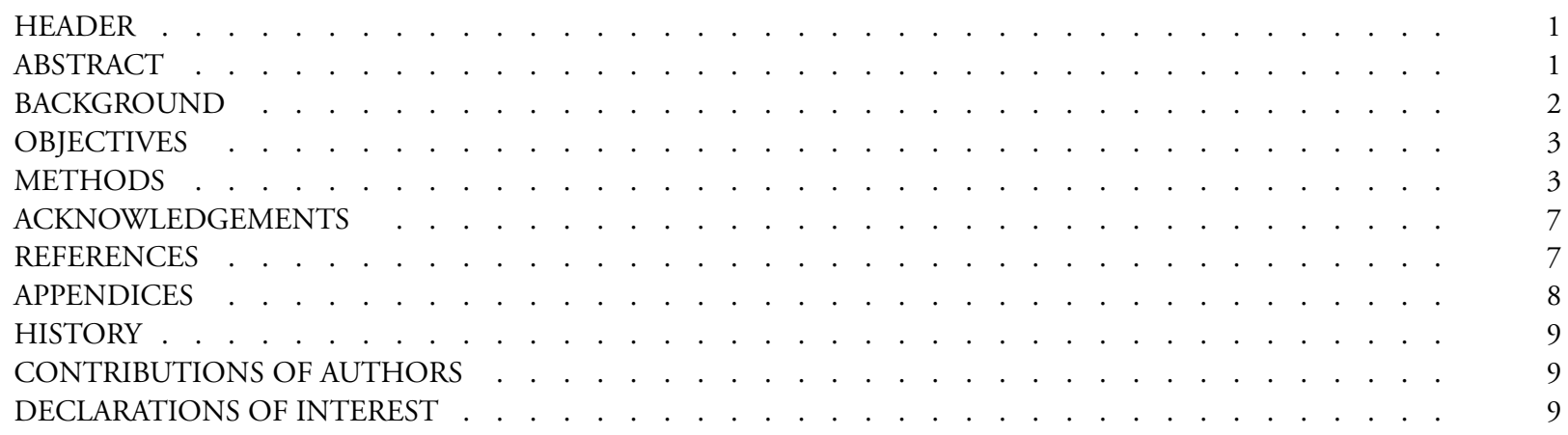




\title{
Patients record systems: effects on dental practice and patient oral health outcomes
}

\author{
Mona Nasser ${ }^{1}$, Zbys Fedorowicz ${ }^{2}$, Tim Newton ${ }^{3}$, Chris Van Weel ${ }^{4}$, Jaap J van Binsbergen ${ }^{5}$, Floris A Van de Laar ${ }^{6}$ \\ ${ }^{1}$ Department of Health Information, Institute for Quality and Efficiency in Health Care, Cologne, Germany. ${ }^{2}$ UKCC (Bahrain \\ Branch), Ministry of Health, Bahrain, Awali, Bahrain. ${ }^{3}$ Division of Health and Social Care Research, KCL Dental Institute, London, \\ UK. ${ }^{4}$ Department of Primary and Community Care, Radboud University Medical Centre, Nijmegen, Netherlands. ${ }^{5}$ Department of \\ General Practice, University Medical Centre, Nijmegen, Netherlands. ${ }^{6}$ Department of Primary and Community Care, 117 HAG, \\ Radboud University Nijmegen Medical Centre, Nijmegen, Netherlands
}

Contact address: Mona Nasser, Department of Health Information, Institute for Quality and Efficiency in Health Care, Dillenburger Street, 27, D-51105, Cologne, D-51105, Germany. mona.nasser@iqwig.de.

Editorial group: Cochrane Effective Practice and Organisation of Care Group.

Publication status and date: New, published in Issue 7, 2010.

Citation: Nasser M, Fedorowicz Z, Newton T, Van Weel C, van Binsbergen JJ, Van de Laar FA. Patients record systems: effects on dental practice and patient oral health outcomes. Cochrane Database of Systematic Reviews 2010, Issue 7. Art. No.: CD008606. DOI: 10.1002/14651858.CD008606.

Copyright (C) 2010 The Cochrane Collaboration. Published by John Wiley \& Sons, Ltd.

\begin{abstract}
A B S T R A C T
This is the protocol for a review and there is no abstract. The objectives are as follows:

The objective of the review is to evaluate the impact of different patient record systems on dental practice and patient care.

We would consider any comparison between different patient record systems against each other but also specifically consider the following questions:

1) Is there any measurable difference between computerized patient record systems and paper based ones?

2) Is there any measurable difference between patient held record systems and practice held ones?

3) Is there any measurable difference between multidisciplinary patient record systems (integrated medical and dental ones) and separate ones?

We also intend to identify and report features of the setting that could potentially affect the impact of the intervention in a specific setting.
\end{abstract}




\section{B A C K G R O U N D}

The problems of dental care are not limited to the efficacy of different dental treatment but also raise questions on how oral health services are organized to deliver the best possible health care. Despite the importance of these topics, only a limited number of high quality systematic reviews have addressed this question (Nasser 2008)

Dental services deal with different kinds of information ranging from details of patient medical and dental history to administration and billing information. Comprehensive and accurate information along with effective and appropriate information exchange and communication between different healthcare professionals and specialties is important to ensure good patient care. Regular, appropriate and adequate information exchange between different specialties and healthcare delivery sectors is of utmost importance considering the increasing amount of complex treatment being undertaken e.g. implant supported dentures which might necessitate the involvement of different dental specialists and in certain situations e.g. elderly people, where the dentist might need to have regular consultation with relevant physicians.

Dentists gather information on the patient medical and dental history profiles along with diagnosis and laboratory information and record the treatment planning process to support clinical decision making. Billing and administrative information are also collected in a dental practice and support the dentists in the management of appointments for the dental practice and the finances of the practice (Shelley 2007). Increasing numbers of dental healthcare providers utilise administrative support systems to keep attendance records of their patients and to facilitate exchange of information with dental technicians and other relevant sectors (Heid 2002).

In summary, different record systems in dental practices collect different sets of information to (a) support dental care and clinical decision making (Haughney 1998; Shelley 2007), (b) improve communication and data exchange in- and outside the dental practice (Shelley 2007), (c) improve the involvement of the patient in clinical decision making and influence patient related outcomes from dental treatment, (d) support the planning, management and auditing of dental health services (Shelley 2007), (e) support administrative (e.g. management of appointments) and financial planning (e.g. billing) (Shelley 2007; Langabeer 2008), (f) ensure applicability of data in research projects (Leake 2005;Langabeer 2008), (g) to support education programs of dental students in dental schools(Langabeer 2008) and (h) improve patient safety (Langabeer 2008). The aspect defined in option ( $\mathrm{g}$ ) is not considered in this review.

\section{Electronic patients records (EPR)}

As a result of improvements in information technology, computers are integrated into the every day life and work of individuals which includes increased usage in clinical and dental practices. Effective use of information technology is also considered to be an important measurement for standards of excellence in clinical practice
(Atkinson 2002; Shelley 2007). A study in 2000 has shown that $85.1 \%$ of all dentists in the United States use computers in their dental practice but most of them use them for administrative reasons and billing and a study in 2006 has shown that only $25 \%$ of all of the dentists use computers in their clinical environment (Atkinson 2002; Schleyer 2007). The electronic patients record (EPR) or computer based medical record aim to facilitate patients' record documentation and simplify the transfer of information between clinical practices. The rationale is to decrease the use of paper, facilitating the collection and retrieving of patient information and ensure comprehensiveness. It is assumed that the paperless system would improve patient care by providing more comprehensive and accurate information on the medical and dental history of an individual and provide the opportunity to exchange the data more easily between practices. The EPR system is supposed to provide facilities in making comparative analysis between group of patients and provide aids in decision making about the diagnosis and treatment of the patients by relating them to clinical guidelines or providing decision support systems for administrative functioning (Atkinson 2002). Nowdays, it not only provides an electronic format of the patient records but also links with a number of diagnostic instruments like intra-oral cameras, digital radiographic systems and CAD-CAM systems (Wagner 2008). However, there were concerns raised whether the confidentiality of the patient record systems would be endangered. The changes which have taken place in computer and software systems has also introduced challenges to dentists to adapt and update their systems or connect them with the new digital or electronic diagnostic systems. The previous experiences with physicians and family practitioners have also shown difficulties for them in adapting themselves with electronic health records due to miscommunication and conflict (Crosson 2005).

Informing patients and sharing the necessary information on their health status and available treatment options with them is now considered an important part of empowering patients to improve the doctor-patient communication. This led to patient held records (PHRs) in some healthcare systems and more recently shared electronic records in which patients keep a complete record of their medical and dental records (Jones 1999; Greenhalgh 2008).

\section{Health information systems}

The data from patient records are not only used individually by dentists to evaluate their dental practice but also collectively to evaluate the quality of clinical care or planning by decision makers. In 1974, the US Department of Health, Education and Welfare requested that the acute hospital discharges cases that were paid by the Medicare or Medicaid would be submitted to them electronically. This resulted in a need to develop administrative databases for medical and dental patient records. These vast amounts of healthcare information provided the opportunity to evaluate health care utilization, outcomes of care and to develop 
health status indicators (Leake 2005).

More recently, the implementation of national and regional health information systems with universally accessible electronic patient records has been discussed or introduced in different countries. Despite the potential of an integrated system to improve the safety and efficiency of clinical care, the large scale structure and complexity of this system introduced new challenges and problems that are not necessarily easily solvable. The environmental, economic, political and technological context of the country can influence the outcome of the health information systems on clinical care and patient outcomes (Greenhalgh 2008). These systems have not only been implemented in high income countries but several projects have explored their effectiveness and applicability in developing countries like the Mosoriot Medical Record System, a joint project of the Indiana University School of Medicine and Moi University School of Medicine in the primary care system in Kenya (Fraser 2005).

\section{Why it is important to do this review}

Several systematic reviews have addressed the general questions on the effectiveness of electronic patients record in improving medical or health care or eHealth in general in improving the quality of health (Uslu 2008; Poissant 2005; Häyrinen 2008). However, we are not aware of any systematic review addressing specifically the question of the effectiveness of patient record systems in dental practices.

None of these reviews discuss the potential relevance of their conclusion to dental practice. This has added complexity to the implementation of the results of these reviews into dental practice due to the differences between dental and medical practice and the current separation of these two systems.

The World Health Organization (WHO) recognizes oral health as part of the general health and priorities programs that leads to integrating these two health care areas, however, despite this recognition in most of the countries the medical and dental practice system are dealt with separately and rarely communicate and exchange information (Din 2008).

Therefore, we decided to undertake a systematic review focusing on the effectiveness of patient record systems implemented in the dental practice on patient care outcomes.

\section{O B JE C T IVES}

The objective of the review is to evaluate the impact of different patient record systems on dental practice and patient care.

We would consider any comparison between different patient record systems against each other but also specifically consider the following questions:
1) Is there any measurable difference between computerized patient record systems and paper based ones?

2) Is there any measurable difference between patient held record systems and practice held ones?

3) Is there any measurable difference between multidisciplinary patient record systems (integrated medical and dental ones) and separate ones?

We also intend to identify and report features of the setting that could potentially affect the impact of the intervention in a specific setting.

\section{METHODS}

\section{Criteria for considering studies for this review}

\section{Types of studies}

The study designs included in this review are described in the Cochrane Effective Practice and Organisation of Care Group (EPOC) Module:

- Randomised controlled trials (RCT) and controlled clinical trials (CCT);

- Controlled before and after studies (CBT) if there are at least two intervention and two control sites and study and control groups are comparable;

- Interrupted time series analyses (ITS) in the case that the point in time when the intervention occurred is clearly defined and there are at least three data points before and three after the intervention.

\section{Types of participants}

1) Dental practice, dental clinic or hospital (e.g. to evaluate the impact of the intervention on quality of care in a dental hospital). 2) Health professionals who are completing the form. This will include both qualified health professionals (those having licensure) or individuals under training.

3) Patients attending dental practices for different oral healthcare problems.

\section{Types of interventions}

Studies that compare the use of any kind of patient record system with another kind (or a component of it with another one) in any dental care setting (in hospital, community or primary care setting). We include all relevant interventions including systematic and multidisciplinary care records (medical and dental records), records in paper and electronic formats or records for specific aspects of oral health care like endodontics and also patient held records. This can include the following comparisons: 
(1) Paper based patient dental records versus electronic ones.

(2) Patient held dental records versus records held by the dentists.

(3) Multidisciplinary care records (integrated medical and dental records) in a primary care system versus separately held records in individual practices.

(4) Electronic patient dental records linked with other diagnostic and treatment tools in a dental office versus electronic patient dental records that are not linked.

(4) Patient dental records that can be shared between different dental practices, clinics and dental laboratories and those that can not be shared.

(5) Electronic patient dental records that can be accessed online by the patient outside the practice and those which can not.

(6) Comparing different patient dental records with different content and structures.

(7) Electronic patient record systems linked with a clinical decision making support system and those which are not.

We will exclude systems designed for dental education unless they are applied to real patient care.

We will also exclude studies undertaken in a testing environment e.g. usability testing of the records without patients or studies that use dental records to identify dead people (forensic dentistry or identification of dead people in disasters).

\section{Types of outcome measures}

\section{Primary outcomes}

We will consider the effectiveness of different record systems or different components in similar records systems on any objective measures of provider performance or patient outcomes.

We will explore whether the outcomes can be considered in any of the following broad categories:

1) Quality of dental care and the management of dental health services e.g. evaluating the timeliness and completeness of restorative care following root canal therapy (Shelley 2007)

2) Patient relevant outcomes e.g. patient satisfaction or clinical outcomes like DMFT. In some interventions like patient held record systems that aim to improve the involvement in their care process, we would also expect outcomes like scales that evaluate patients' perception of level of control over decision making during the consultation will be also used.

3) Improving communication and data exchange between dental settings and other related settings e.g. some practices might use joint medical and dental records to ensure that both the dentists and physician are adequately aware of the important health problem of their patients.

Outcomes on improving communication and data exchange include a) improving data exchange between different dental practices or departments in dental school or dental hospital, b) improving data exchange between the dental and medical depart- ment and c) improving data exchange between the dental practice and dental and medical laboratories.

4) Financial and scheduling outcomes e.g. number of bill complaints or rebills.

We will not include studies that only evaluate the effectiveness of the program on subjective measures of provider performance as they are subject to bias unless explicit robust and validated measures are used (Adams 1999).

\section{Secondary outcomes}

Process outcomes of the work of the software like quality of data, availability of data, accessibility to data, ease of production of data, presentation of data and handling data.

\section{Search methods for identification of studies}

\section{Electronic searches}

See: Effective Practice and Organisation of Care (EPOC) Group methods used in reviews.

The Database of Abstracts of Reviews of Effectiveness (DARE) will be searched for related reviews.

The following electronic databases will be searched for primary studies:

(a)The EPOC Specialised Register (and the database of studies awaiting assessment) will be reviewed (see SPECIALISED REGISTER under GROUP DETAILS).

(b)The Cochrane Central Register of Controlled Trials (CENTRAL).

(c)Bibliographic databases, including MEDLINE, EMBASE, and CINAHL.

Electronic databases will be searched using a search strategy developed incorporating the methodological component of the EPOC search strategy (using the EPOC definitions - RCT, CCT, CBA, ITS) combined with selected MeSH terms and free text terms relating to electronic record systems. This search strategy will be translated into the other databases using the appropriate controlled vocabulary as applicable. We will not use language restrictions.

\section{Language}

There will be no language restrictions on included studies and we will arrange to translate and report any relevant non-English papers.

\section{Searching other resources}

(d) Handsearching of those high-yield journals and conference proceedings which have not already been handsearched on behalf of the Cochrane Collaboration.

(e) Reference lists of all papers and relevant reviews identified.

(f) Authors of relevant papers will be contacted regarding any further published or unpublished work. 
(g) Authors of other reviews in the field of effective professional practice will be contacted regarding relevant studies of which they may be aware.

(h) We will search ISI Web of Science for papers which cite studies included in the review.

\section{Data collection and analysis}

\section{Selection of studies}

Two review authors ( $\mathrm{MN}$ and $\mathrm{ZF}$ ) will independently read the titles and/or abstracts resulting from the search process and eliminate any obviously irrelevant studies. The remaining studies classified as clearly relevant or unclear will be retrieved in full text. The same two review authors will independently assess these for inclusion. Difference in opinion that cannot be resolved by consensus between the two review authors will be discussed with the third review author (TJN). After assessment, the review authors will eliminate from further review any remaining trials that do not match the inclusion criteria and note the reasons for their exclusion in the 'Characteristics of excluded studies' table.

\section{Data extraction and management}

Two review authors will enter details of the included studies in the 'Characteristics of included studies' table in RevMan 5 and cross-check details. The following data will be extracted from the studies as recommended in the EPOC Module (Bero 2009).

1. Methods of the study: study design, methodological quality, unit of allocation, unit of analysis, study power.

2. Participants: The characteristics of the people in the dental practice or clinic responsible for data entry, uses the data or is affected by the system (dentists, nurses, patients and etc), level of training, profession, clinical speciality, age, time since graduation/years of practice, number of subjects (or clusters) included in the study, episode of care.

\section{Interventions:}

(a) the format and type of the record system;

(b) the characteristics or components of the record system;

(c) the setting and environment in which the record system was implemented;

(d) a definition whether the record system is a stand alone program or part of a wider and larger program (e.g. national, regional or institutional health information system) stand alone intervention or a component of a wider program like an integrated dental and medical records system as part of a wider strategy to integrate dental and medical care;

(e) the purpose of the installing the record system, the people who are working with the record system;

(f) level of implementation e.g. implemented in a dental practice or a health care system.
4. Outcomes: description of the main outcomes measures, length of time outcomes measured after initiation of the intervention, possible ceiling effect e.g. little room for improvement in provider performance because it was adequate without the intervention (based on baseline measurements or control group performance). 5. Dental services setting: individual private practice, primary care setting, dental clinic, hospital and etc.

6. Results: RCTs and CCTs: The results for the main outcomes in natural units. The baseline performance and post-intervention differences between study and control groups (including statistical significance if reported; and indicating if the units of randomisation and analysis were different).

CBAs: The results for the main outcomes in natural units. The baseline difference in the pre-post intervention change between groups. For each available comparison, the difference across study groups of the pre-post intervention change (including statistical significance if reported; and in all cases reporting a more favourable provider/patient outcome in the intervention group as a positive finding i.e. where differences in the groups are in the intended direction)

ITSs: The results for the main outcomes in natural units (in all cases reporting a more favourable provider/patient outcome attributable to the intervention as a positive finding i.e. where changes in the outcome are in the intended direction).

7. Source of funding and ethical approval.

\section{Assessment of risk of bias in included studies}

Assessment of methodological quality will be based on EPOC guidelines. The quality of all eligible studies will be assessed by two independent review authors (MN and ZF) using criteria described in the EPOC module.

Each review author will grade the studies using a modified version of the risk of bias table that was designed to replace quality criteria in the EPOC data collection checklist (EPOC 2009). . We will compare the evaluations and discuss and resolve any inconsistencies between the review authors in the interpretation of inclusion criteria and their significance to the selected.

We will assess the following domains as 'Yes' (i.e. low risk of bias), 'Unclear' (uncertain risk of bias) or 'No' (i.e. high risk of bias) for studies with a separate control group (RCTs, CCTs, CBAs):

1. Was the allocation sequence adequately generated?

2. Was the allocation adequately concealed?

3. Were baseline outcome measurements similar?

4. Were baseline characteristics similar?

5. Were incomplete outcome data adequately addresses?

6. Was knowledge of the allocated interventions adequately prevented during the study?

7. Was the study adequately protected against contamination?

8. Was the study free form selective outcome reporting?

9. Was the study free from other risks of bias?

We will assess the following domains as 'Yes' (i.e. low risk of bias) and 'No' (i.e. high risk of bias) for interrupted time series studies: 
1. Was the intervention independent from other changes?

2. Was the shape of the intervention effect pre-specified?

3. Was the intervention unlikely to affect data collection?

4. Was the knowledge of the allocated interventions adequately prevented during the study?

5. Were incomplete outcome data adequately addressed?

6. Was the study free from selective outcome reporting?

7. Was the study free form other risk of bias?

Based on this assessment, we will group the included studies to: (A) Low risk of bias (plausible bias unlikely to seriously alter the results)

(B) Moderate risk of bias (plausible bias that raises some doubt about the results)

(C) High risk of bias (plausible bias that seriously weakens confidence in the results)

Any discrepancies in quality ratings will be resolved by discussion and involvement of the third author, if needed.

\section{Evaluating the external validity in the included studies}

We will evaluate the external validity of the included studies using the external validity tool suggested by Green 2006 . The tool evaluates the external validity and looks at three aspects "reach and representativeness", "implementation and consistency of effects" and "maintenance and institutionalization" Appendix 1. We will use the result of the evaluation to provide guidance for decision makers and practitioners to judge about the generalizibility of the results of this systematic review to their local setting.

\section{Dealing with missing data}

We will make attempts to retrieve missing data from the investigators for any of the included studies and, if unsuccessful or the discrepancies are significant, we will provide a narrative synthesis of the data as reported.

\section{Assessment of heterogeneity}

We will assess clinical heterogeneity by:

1) Using a logical and common sense approach: examining the characteristics of the studies, the similarity between the types of participants, the interventions and the outcomes as specified in the criteria for included studies. In view of the expectation of a degree of clinical heterogeneity between the studies we intend using the random-effects model with studies grouped by action.

2) Statistical heterogeneity will be assessed using a Chi ${ }^{2}$ test and the $\mathrm{I}^{2}$ statistic, where $\mathrm{I}^{2}$ values over $50 \%$ indicate moderate to high heterogeneity (Higgins 2003).

3) Visual examinations of graphs for outliers and between study differences.

\section{Assessment of reporting biases}

If sufficient homogenous data are available to assess publication bias we will follow the recommendations on testing for funnel plot asymmetry as described in section 10.4.3.1 of the Cochrane Handbook for Systematic Reviews of Interventions 5.0.0 (Higgins 2008) and we will explore these in the discussion if appropriate.

\section{Data synthesis}

Two review authors (MN and ZF) will analyse the data and report them as specified in Chapter 9 of the Cochrane Handbook for Systematic Reviews of Interventions Version 5.0.0 (Higgins 2008) and the EPOC checklists (http://www.epoc.cochrane.org/ en/handsearchers.html)

If studies are sufficiently similar a statistical analysis will be performed. If studies are so heterogenous that statistical combination is not possible, a narrative presentation of relevant data will be presented in tables or in forest plots without the summary statistics. We will report outcomes in natural units. If baseline measurements are reported, we will report pre-interventions and post-interventions means or proportions for both study and control group and will calculate the unadjusted and adjusted (for any baseline imbalance) absolute change from baseline with $95 \%$ confidence limits. For dichotomous outcome measures, we will present both risk differences and relative risk reductions.

For ITS, we will report the level of outcomes immediately after the implementation of the intervention and the changes in the slopes of the regression lines before and after the implementation of the intervention. If the method of analysis was inappropriate, time series regression will be used to re-analyse each comparison where possible (Ramsey 2003).

For cluster RCTs and CCTS, we will evaluate whether the included studies have unit of analysis problems or not. If this would be the case, we will either try to obtain a correct analysis or reduce the size of the patient RCT to take into account the clustering effect. To do this, we will estimate the design effect using the average cluster size and the intracluster correlation (ICC) if this data can be obtained from the paper.

The analysis will be based upon dichotomous outcomes measures. If more than one measure is reported for each endpoint, we will use the primary measure as defined by the investigator of the study. If there is not clear primary measure available, we will calculate and use a median from all available measures.

When a summary measure of effect can't be computed, due to the expected heterogeneity in the studies, we will consider reporting the following information across all comparisons:

- the number of comparison showing a positive direction of effect;

- the median effect size across all comparisons;

- the median effect size across comparisons without unit of analysis errors; 
- inter-quartile ranges of effect sizes across included comparisons;

- range of effect sizes across all comparisons;and

- the number of comparisons showing statistically significant effects.

Continuous outcomes will be used for secondary analysis and further interpretation of findings.

\section{Subgroup analysis and investigation of heterogeneity}

If data are available, we will compare the effect sizes across record systems according to potential effect modifiers. The main poten- tial co-founders would be the source of funding for the trial, the type of setting where the intervention was implemented (in a socioeconomic disadvantaged area or not), targeted healthcare professionals (type of healthcare professional and years in practice).

\section{ACKNOWLEDGEMENTS}

The review authors would like to thank the Cochrane EPOC Group, Emma Tavender, Alain Mayhew and Russell Gruen for their help in conducting this systematic review.

\section{REFERENCE S}

\section{Additional references}

\section{Adams 1999}

Adams AS, Soumerai SB, Lomas J, Ross-Degnan D. Evidence of self-report bias in assessing adherence to guidelines. International Journal for Quality in Health Care 1999;11(3):187-92.

\section{Atkinson 2002}

Atkinson JC, Zeller GG, Shah C. Electronic patient records for dental school clinics: more than paperless systems. Journal of Dental Education 2002;66(5):634-42.

\section{Bero 2009}

Bero L, Deane K, Eccles M, Grimshaw J, Gruen RL, Mayhew A, Oxman AD, Pantoja T, Paulsen E, Shepperd $S$, Tavender E, Zwarenstein M. Cochrane Effective Practice and Organisation of Care Group. About The Cochrane Collaboration (Cochrane Review Groups (CRGs)) 2009; Vol. 1. Art. No.: EPOC.

\section{Crosson 2005}

Crosson JC, Stroebel C, Scott JG, Stello B, Crabtree BF. Implementing an electronic medical record in a family medicine practice: communication, decision making, and conflict. Annals of Family Medicine 2005;4:307-11.

\section{Din 2008}

Din F, Powell V. Integration of medical and dental records to improve healthcare outcomes, costs and overall public health. Change.gov Community Healthcare Meeting and Teleconference, December 17, 2008. Robert Morris University, Moon Township, PA.

\section{EPOC 2009}

Cochrane Effective Practice and Organisation of Care Review Group. Risk of Bias (replaces quality criteria in the EPOC data collection checklist). http:// epoc.cochrane.org/sites/epoc.cochrane.org/files/uploads/ Risk\%20of\%20Bias\%2005-01-2009.doc [Last access data: 26.05.2010]

\section{Fraser 2005}

Fraser HS, Biondich P, Moodley D, Choi S, Mamlin BW, Szolovits P. Implementing electronic medical record systems in developing countries. Informatics in Primary Care 2005; 13(2):83-95.

\section{Green 2006}

Green LW, Glasgow RE. Evaluating the relevance, generalization, and applicability ofresearch: issues in external validation and translation methodology. Evaluation and the Health Professions 2006;29(1):126-53.

\section{Greenhalgh 2008}

Greenhalgh T, Stramer K, Bratan T, Byrne E, Mohammad $\mathrm{Y}$, Russell J. Introduction of shared electronic records: multi-site case study using diffusion of innovation theory. BMJ 2008;337:a1786. [DOI: 10.1136/bmj.a1786]

\section{Haughney 1998}

Haughney MG, Devennie JC, Macpherson LM, Mason DK. Integration of primary care dental and medical services: a three-year study. British Dental Journal 1998;184(7): 343-7.

\section{Heid 2002}

Heid DW, Chasteen J, Forrey AW. The electronic oral health record. Journal of Contemporary Dental Practice 2002; 3(1):43-54.

\section{Higgins 2003}

Higgins JP, Thompson SG, Deeks JJ, Altman DG. Measuring inconsistency in meta-analyses. BMJ 2003;327 (7414):557-60.

\section{Higgins 2008}

Higgins JPT, Green S (editors). Cochrane Handbook for Systematic Reviews of Interventions Version 5.0.0 [updated February 2008]. The Cochrane Collaboration, 2008.

\section{Häyrinen 2008}

Häyrinen K, Saranto K, Nykänen P. Definition, structure, content, use and impacts of electronic health records: a review of the research literature. International Journal of Medical Informatics 2008;77(5):291-304.

Jones 1999

Jones R, McConville J, Mason D, Macpherson L, Naven L, McEwen J. Attitudes towards, and utility of, an integrated 
medical-dental patient-held record in primary care. British Journal of General Practice 1999;49(442):368-73.

\section{Langabeer 2008}

Langabeer JR 2nd, Walji MF, Taylor D, Valenza JA.

Economic outcomes of a dental electronic patient record.

Journal of Dental Education 2008;72(10):1189-200.

Leake 2005

Leake JL, Werneck RI. The use of administrative databases to assess oral health care. Journal of Public Health Dentistry 2005;65(1):21-35.

\section{Nasser 2008}

Nasser M, Fedorowicz Z, Bastian H. Comparing the priorities in oral health with the existing evidence in the Cochrane Library. German Journal of Evidence and Quality in Health Care. 2008; Vol. 102 Suppl. 6:S 94-95.

\section{Poissant 2005}

Poissant L, Pereira J, Tamblyn R, Kawasumi Y. The impact of electronic health records on time efficiency of physicians and nurses: a systematic review. Journal of the American Medical Informatics Association 2005;12(5):505-16.

Ramsey 2003

Ramsey CR, Matowe L, Grilli R, Grimshaw JM, Thomas RE. Interrupted time series designs in health technology assessment: lessons from two systematic reviews of behavior change strategies. International Journal of Technology Assessment in Health care 2003;19(4):613-23.

\section{Schleyer 2007}

Schleyer T, Spallek H, Hernández P. A qualitative investigation of the content of dental paper-based and computer-based patient record formats. Journal of the American Medical Informatics Association 2007;14(4): 515-26.

\section{Shelley 2007}

Shelley PQ, Johnson BR, BeGole EA. Use of an Electronic Patient Record system to evaluate restorative treatment following root canal therapy. Journal of Dental Education 2007;71(10):1333-9.

\section{Uslu 2008}

Uslu AM, Stausberg J. Value of the electronic patient record: an analysis of the literature. Journal of Biomedical Informatics 2008;41(4):675-82.

Wagner 2008

Wagner IV, Ireland RS, Eaton KA. Digital clinical records and practice administration in primary dental care. British Dental Journal 2008;204(7):387-95.

* Indicates the major publication for the study

\section{A P P E N D I CES}

\section{Appendix I. Proposed quality criteria to evaluate the external validity of clinical trials}

\section{Reach and representativeness}

A. Participation: Are there analyses of the participation rate among potential (a) settings, (b) delivery staff, and (c) patients (consumers)?

B. Target audience: Is the intended target audience stated for adoption (at the intended settings such as worksites, medical offices, etc.) and application (at the individual level)? C. Representativeness-Settings: Are comparisons made of the similarity of settings in study to the intended target audience of program settings-or to those settings that decline to participate?

D. Representativenes-Individuals: Are analyses conducted of the similarity and differences between patients, consumers, or other subjects who participate versus either those who decline, or the intended target audience?

\section{Program or policy implementation and adaptation}

A. Consistent implementation: Are data presented on level and quality of implementation of different program components?

B. Staff expertise: Are data presented on the level of training or experience required to deliver the program or quality of implementation by different types of staff?

C. Program adaptation: Is information reported on the extent to which different settings modified or adapted the program to fit their setting?

D. Mechanisms: Are data reported on the process(es) or mediating variables through which the program or policy achieved its effects?

III. Outcomes for decision making 


\begin{abstract}
A. Significance: Are outcomes reported in a way that can be compared to either clinical guidelines or public health goals?

B. Adverse consequences: Do the outcomes reported include quality of life or potential negative outcomes?

C. Moderators: Are there any analyses of moderator effects-including of different subgroups of participants and types of intervention staff-to assess robustness versus specificity of effects?

D. Sensitivity: Are there any sensitivity analyses to assess dose-response effects, threshold level, or point of diminishing returns on the resources expended?

E. Costs: Are data on the costs presented? If so, are standard economic or accounting methods used to fully account for costs?

IV. Maintenance and institutionalization

A. Long-term effects: Are data reported on longer term effects, at least 12 months following treatment?

B. Institutionalization: Are data reported on the sustainability (or reinvention or evolution) of program implementation at least 12 months after the formal evaluation?

C. Attrition: Are data on attrition by condition reported, and are analyses conducted of the representativeness of those who drop out?

Reference: Table 3 (Page 137) (Green 2006).
\end{abstract}

\title{
H I S T O R Y
}

Protocol first published: Issue 7, 2010

\section{CONTRIBUTIONS OFAUTHORS}

$\mathrm{MN}$ is responsible for organizing the retrieval of papers; writing to authors of papers for additional information; obtaining and screening data on unpublished studies.

$\mathrm{MN}$ and ZF are responsible for screening search results; screening retrieved papers against inclusion criteria; appraising the quality of papers; data collection for the review; extracting data from papers.

$\mathrm{MN}$ is responsible for entering the data into RevMan and analysis of the data.

All review authors (MN, ZF and Tim J Newton (TJN)) are responsible for interpreting data and contribute to writing the protocol and review.

$\mathrm{MN}$ is responsible for co-ordinating the review, conceived the idea for the review and is the guarantor for the review.

\section{DECLARATIONS OF INTEREST}

There are no financial conflicts of interest and the review authors declare that they do not have any associations with any parties who may have vested interests in the results of this review. 